\title{
Multiparametric MRI Fusion-guided Biopsy for the Diagnosis of Prostate Cancer: Results of the First 100 Consecutive Patients
}

\author{
D Güven Aslan \\ Dokuz Eylül University Faculty of Medicine, Department of Urology, Izmir, Turkey
}

\begin{abstract}
Objective: Our aim was to compare prostate cancer detection rates according to International Society of Urological Pathology (ISUP) grade group (GG) among magnetic resonance imaging (MRI)-targeted biopsies (TBX), systematic biopsies (SBx) and a combination of both (TBx + SBx).

Materials and Methods: Between July 2018 and Nov 2020, 100 patients undergoing MRI-targeted prostate biopsy and SB were analysed retrospectively. Men with MRI-visible prostate lesions underwent both MRI-targeted (UroNavi) and systematic 12-core biopsy. The primary outcome was cancer detection according to ISUP GG. The secondary outcome were detection of cancers of GG 2 or higher.

Results: The overall cancer detection rate in the study was 43\%. Among these, a histopathological ISUP grade of 1 was detected in $44.2 \%$, ISUP grade 2 in $32.5 \%$ and higher-grade groups in $23.2 \%$ of patients. MRI-TB detected 38 and SBx biopsy detected 30 of the overall study cohort. Combined biopsy led to a cancer diagnoses in 18 more men than with either method alone. Significant cancer detection was 53.4\% for TBx, 39.5\% for SBx, and highest 55.8\% for combination $\mathrm{TBx}+\mathrm{SBx}(\mathrm{p}=0.038)$. Most of the cancers missed by SBx were clinically significant. No significant side effects were recorded. The overall cancer detection rate was $20.4 \% / 46.6 \%$ and $90.2 \%$ for prostate imaging reporting and data system $3 / 4 / 5$, respectively.

Conclusion: Our results have shown that among patients with lesions visible on MRI, combined biopsy led to greater detection of all prostate cancers, providing increased detection of clinically significant tumours.

Keywords: Prostate cancer, detection, targeted biopsy, MRI US Fusion, prostate biopsy
\end{abstract}

\section{Introduction}

Prostate cancer ( $\mathrm{PCa}$ ) is the most common cancer among Northern and Western European men (1). To further improve survival in patients with $\mathrm{PCa}$, it is necessary to differentiate between clinically insignificant and clinically significant cancer. In current clinical practice, Transrectal Ultrasound-guided prostate Biopsy (TRUS $\mathrm{Bx}$ ) is the commonly used technique to further evaluate a suspected PCa diagnosis. The current diagnostic approach including PSA testing and digital rectal examination followed by transrectal ultrasound biopsies lacks both sensitivity and specificity in PCa detection and offers limited information about the aggressiveness and stage of the cancer $(2,3,4)$. Recent scientific work supports the rapidly growing use of multiparametric magnetic resonance imaging (mpMRI) as a promising tool of growing importance in PCa evaluation. With the introduction of mpMRI, the accuracy for the localisation and detection of PCa is improved $(3,5,6)$. Its use may improve many aspects of PCa management, from initial detection of significant tumours using mpMRI-guided biopsies to evaluation of biological aggressiveness and accurate staging, which can facilitate appropriate treatment selection. The use of mpMRI and MRI-targeted biopsy (TB) has been shown to improve PCa detection by increasing the overall PCa detection rate and reducing the detection of insignificant tumours $(3,4,5,6,7)$. However, experience with MRI-targeted biopsies in $\mathrm{PCa}$ diagnosis in Turkey has been very limited. Therefore, we carried out this study to evaluate the use of mpMRI in disease detection and the assessment of histopathological aggressiveness.

\section{Materials and Methods}

Based on our prospective database, 100 consecutive biopsy-naive patients who underwent MRI-TB combined with systematic 12core prostate biopsy $(\mathrm{TBx}+\mathrm{SBx})$ during the period between July 2018 and November 2020 were identified and included in the 
analysis. Patients with firm palpable prostate nodules suggestive of cancer, any patient who had previously undergone prostate biopsy and patients with known urinary tract infections were excluded from the study. All patients gave informed consent. Patients for whom had complete pathological data were available for each $B x$ scheme were investigated. The patient's age and PSA level and the highest GS of each Bx scheme were evaluated. Since the study was a retrospective case-control study, IRB approval was not required.

All mpMRI for targeted biopsies were reviewed by the specified radiologist. In patients with a prostate imaging reporting and data system (PI-RADS) lesion $\geq 3$ (according to the PI-RADS" classification) (8), software-based MRI-targeted ultrasound fusion biopsies using UroNav® (Invivo Corp, Philips, USA) were conducted. All biopsies were performed using a transrectal approach under IV sedation anaesthesia and antibiotic prophylaxis with a single dose of Fosfomycin the night before and IV Gentamycin $120 \mathrm{mg}$ + third Generation Cephalosporin 1 hour before the procedure. MRI image- fusion-targeted biopsies were taken from each target, and at least 2 core samples were taken from each target lesion. In addition to targeted biopsies, SBx were performed using a 12-core approach. The biopsy cores were evaluated by dedicated uro-pathologists. The biopsy Gleason score was defined as the highest Gleason score in at least one core and was reported using the International Society of Urological Pathology (ISUP) Consensus Conference 2014 grading system (9).

\section{Statistical Analysis}

Descriptive statistics included continuous variables and frequencies and proportions for categorical variables. Differences were analysed with the use of a chi-square test using SPSS software. Differences were considered statistically significant at $\mathrm{p} \leq 0.05$.

\section{Results}

Patient demographics of the study cohort stratişed by biopsy approach are depicted in Table 1. The median number of TB cores sampled per region of interest was $4(2,3,4,5,6,7)$.

The overall cancer detection rate in the study was $43 \%$. Among these 43 patients, a histopathological ISUP grade of 1 was detected in 19 (44.2\%) patients, ISUP grade 2 in 14 (32.5\%) patients and higher-grade groups in 10 patients (23.2\%). Cancer was detected by MRI/US-TB in 38 and by SB in 30 of the overall study cohort of 100 patients. Combined biopsy led to cancer diagnoses in 18 more men than with either method alone. There was a significant difference in the missed cancer ratio between TBx and SBx (11.6\% vs $30.2 \%, p=0.01)$, in that any grade of cancer detection by SBx alone was significantly lower than by TBx alone.

Many patients had different PI-RADS scores registered by target lesion in the same patient. When the results were divided according to PIRAD grade, the positivity rates were $20.4 \%$, $46.6 \%$ and $90.2 \%$ for PIRAD 3, PIRAD 4 and PIRAD 5 scores, respectively.

The rate of significant cancer detection was $53.4 \%$ for TBx, $39.5 \%$ for $\mathrm{SBx}$ and $55.8 \%$ for combination $\mathrm{TBx}+\mathrm{SBx}$, indicating that more significant cancer (ISUP grade 2 or higher) is detected by MRI fusion biopsy. If only SBx biopsies had been performed, $13.9 \%$ of clinically significant cancers would have been misclassified. Most of the cancers missed by SBx were clinically significant (Figure 1).

No significant side effects such as fever, urinary retention, urosepsis, and hospitalisation were recorded in any patient, except mild haematuria and hematospermia.

\section{Discussion}

In modern times, mpMRI of the prostate is becoming an integrative part of the diagnostic workup of PCa. Several prospective trials demonstrated that $\mathrm{TBx}$ can increase $\mathrm{PCa}$

\begin{tabular}{|c|c|c|c|c|c|}
\hline Variables & & \multicolumn{3}{|c|}{$\begin{array}{l}\text { Combined MRI-TBx + SBx method } \\
(n=100)\end{array}$} & \\
\hline Age (year) & & \multicolumn{3}{|c|}{$58.6 \pm 4.1(48-75)$} & \\
\hline PSA (ng/dL) & & \multicolumn{3}{|c|}{$6.1 \pm 3.9(2.2-27)$} & \\
\hline \multicolumn{2}{|l|}{$\begin{array}{l}\text { Cancer Detected } \\
\mathrm{n}=43\end{array}$} & MRI-TBx & SBx & $\begin{array}{l}\text { Combined } \\
\text { MRI-TBx + SBx }\end{array}$ & - \\
\hline & Missed Cancer & 5 & 13 & 0 & - \\
\hline \multirow{5}{*}{$\begin{array}{l}\text { Biopsy } \\
\text { ISUP } \\
\text { Grades }\end{array}$} & 1 & 15 & 13 & 19 & \\
\hline & 2 & 13 & 9 & 14 & \\
\hline & 3 & 8 & 6 & 8 & \\
\hline & 4 & 1 & 1 & 1 & \\
\hline & 5 & 1 & 1 & 1 & \\
\hline PI-RADS 3 & & & & $20.4 \%$ & \\
\hline PI-RADS 4 & & & & $46.6 \%$ & \\
\hline PI-RADS 5 & & & & $90.2 \%$ & \\
\hline
\end{tabular}




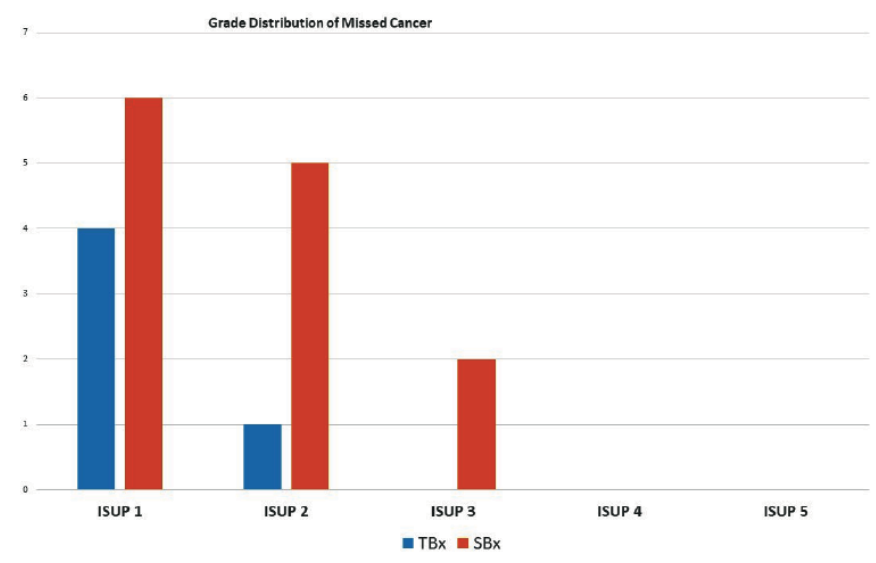

Figure 1. Distributions of ISUP grades of missed cancer according to the biopsy method

ISUP: International Society of Urological Pathology

detection rates, especially that of clinically significant $\mathrm{PCa}$ (GGG $\geq 2$ ), while lowering the detection rate of low-risk PCa $(2,10,11,12,13,14)$.

In this cohort of patients, combined TBx + SBx biopsy provided a more accurate diagnosis than MRI-targeted or SB alone. Consistent with earlier studies, we found higher cancer detection rates on TBx when compared with $\operatorname{SBx}(7,10)$. However, 5 out of 43 prostate cancers $(11.6 \%)$ went undetected by TBx alone. Missed cancers by TBx may reflect the underlying limitation of mpMRI, which is the possibility of invisible PCa on mpMRI, varying inter-reader agreement on the mpMRI results and missing the target lesion in biopsy.

The combination of TB and SB was superior to either method alone for the detection of clinically significant PCa (ISUP 2 or higher). When TBx alone to SBx alone are compared for the detection rate of significant $\mathrm{PCa}$ diagnosis, overall TB alone tends to detect more significant PCa but this superiority does not reach statistical significance. Our result complements previous results favouring MRI-targeted biopsies $(15,16)$. Several other studies have compared MRI fusion-TB with SB with diverging results depending on the type of SB used or number of cores taken. Some found that MRI-TB led to increased detection of high-risk cancer and decreased detection of low-risk cancer but missed up to $6 \%$ of higher-risk tumours. On the other hand, others found similar detection rates in systematic and targeted transrectal biopsies $(10,16,17)$. In the present study, SBx had inferior yet important performance to TBx in determining highgrade cancers. This may be a result of the cognitive fusion bias within the SBX that occurred when the urologist performing the SBX was aware of the localisation of the suspicious lesion on mpMRI. In our study, if a pure TBx strategy omitting SBx were to be applied, this would lead to missing up to 5 patients with cancer. Our results support that to obtain the most accurate assessment of the entire prostate gland, SBx remains necessary, in addition to TBx, due to limitations of mpMRI performance/ reading and of precision during lesion targeting.

In the current study, we report our experience of biopsied PIRADS $3,4,5$ lesions. We found our detection rate of prostatic adenocarcinoma amongst these lesions to be $20.42 \%, 46.6 \%$ and $90.2 \%$ respectively. The results of the current study demonstrate a good correlation between PI-RADS scores and positive biopsy. The cancer detection rate stratified by PI-RADS score is similar to that reported in the published literature (18). However, there are different cancer detection rates according to PI-RADS score. Previous studies reported overall cancer detection rates of PI-RADS v2 categories 3, 4 and 5 of 39\%, 72\% and $91 \%$, respectively, for all prostate cancers. On the other hand, several studies reported lower detection rates (19). The DWI PI-RADS score alone correlates well with positive biopsies in the peripheral zone, but not the transition zone (20). PI-RADS v2 uses a simplified approach but shows a lower diagnostic accuracy. This could lead to a higher rate of false-negative results with the risk of missing tumours within low PI-RADS score levels. When performing a TB, combination with a SB still provides the highest detection rate of prostate cancer.

\section{Study Limitations}

The present study has several limitations. First and foremost, our manuscript was based on a retrospective analysis of only one centre. Our study was limited to a rather small cohort of 100 patients. In all our patients, MRI-TBx was performed by using the transrectal approach. We could not measure the potential positive or negative effects of other platforms on detection rates using transperineally biopsy systems. Moreover, failure of $\mathrm{mpMRI}$ fusion biopsy due to incorrect $\mathrm{mpMRI}$ image registration or mismatching of image planes, inaccurate sampling and intralesion Gleason score heterogeneity may have impacted on our results. However, our data reflect the real-life picture.

\section{Conclusion}

Our study shows that MRI-targeted biopsies in patients with suspected PCa result in a high detection rate and clinical significance of diagnosed tumours. These results suggest that MRI-based diagnosis and subsequent targeted biopsies fulfil an important role in increasing the detection rate and accuracy in the diagnosis of PCa. Since multiparametric MRI still has some risk of missing tumours; additional systematic 12 core biopsies should not currently be omitted.

\section{Acknowledgements}

Publication: The results of the study were not published in full or in part in form of abstracts.

Contribution: There is not any contributors who may not be listed as author.

Conflict of Interest: No conflict of interest was declared by the author.

Financial Disclosure: The author declared that this study received no financial support.

\section{Ethics}

Ethics Committee Approval: Since the study was a retrospective case-control study, IRB approval was not required.

Informed Consent: All patients gave informed consent.

Peer-review: Externally peer-reviewed. 


\section{References}

1. Jemal A, Siegel R, Xu J, Ward E. Cancer statistics, 2010. CA Cancer J Clin 2010;60:277-300.

2. Ahmed HU, El-Shater Bosaily A, Brown LC, et al. Diagnostic accuracy of multi-parametric MRI and TRUS biopsy in prostate cancer (PROMIS): a paired validating confirmatory study. Lancet 2017;389:815-822.

3. Puech $P$, Rouviere $\mathrm{O}$, Renard-Penna $\mathrm{R}$, et al. Prostate cancer diagnosis: multiparametric MR-targeted biopsy with cognitive and transrectal US-MR fusion guidance versus systematic biopsy: prospective multicenter study. Radiology 2013;268:461-469.

4. Pinto PA, Chung PH, Rastinehad AR, et al. Magnetic resonance imaging/ultrasound fusion guided prostate biopsy improves cancer detection following transrectal ultrasound biopsy and correlates with multiparametric magnetic resonance imaging. J Urol 2011;186:1281-1285.

5. Moore CM, Robertson NL, Arsanious N, et al. Image-guided prostate biopsy using magnetic resonance imaging-derived targets: a systematic review. Eur Urol 2013;63:125-140.

6. Siddiqui MM, Rais-Bahrami $\mathrm{S}$, Truong $\mathrm{H}$, et al. Magnetic resonance imaging/ultrasound-fusion biopsy significantly upgrades prostate cancer versus systematic 12-core transrectal ultrasound biopsy. Eur Urol 2013;64:713-719.

7. Bae JH, Kim SH. Transrectal ultrasound-guided prostate biopsy versus combined magnetic resonance imaging-ultrasound fusion and systematic biopsy for prostate cancer detection in routine clinical practice. Ultrasonography 2020;39:137-143.

8. Weinreb JC, Barentsz JO, Choyke PL, et al. PI-RADS Prostate Imaging Reporting and Data System: 2015, version 2. Eur Urol 2016;69:16-40.

9. Epstein JI, Egevad L, Amin MB, et al. The 2014 International Society of Urological Pathology (ISUP) Consensus Conference on Gleason Grading of Prostatic Carcinoma: definition of grading patterns and proposal for a new grading system. Am J Surg Pathol 2016;40:244-252.

10. Siddiqui MM, Rais-Bahrami S, Turkbey B, et al. Comparison of MR/ ultrasound fusion-guided biopsy with ultrasound-guided biopsy for the diagnosis of prostate cancer. JAMA 2015;313:390-397.
11. Diamand R, Oderda M, Al Hajj Obeid W, et al. A multicentric study on accurate grading of prostate cancer with systematic and MRI/US fusion targeted biopsies: comparison with final histopathology after radical prostatectomy. World J Urol 2019;37:2109-2117.

12. Ahdoot M, Wilbur AR, Reese SE, et al. MRI-Targeted, Systematic, and Combined Biopsy for Prostate Cancer Diagnosis. N Engl J Med 2020;382:917-928.

13. Kasivisvanathan V, Rannikko AS, Borghi M, et al. MRI-targeted or standard biopsy for prostate-cancer diagnosis. N Engl J Med 2018;378:1767-1777.

14. Drost FH, Osses DF, Nieboer D, et al. Prostate MRI, with or without MRI-targeted biopsy, and systematic biopsy for detecting prostate cancer. Cochrane Database Syst Rev 2019;4:CD012663.

15. Radtke JP, Kuru TH, Bonekamp D, et al. Further reduction of disqualification rates by additional MRI-targeted biopsy with transperineal saturation biopsy compared with standard 12-core systematic biopsies for the selection of prostate cancer patients for active surveillance. Prostate Cancer Prostatic Dis 2016;19:283-291.

16. Hansen NL, Kesch C, Barrett T, et al. Multicentre evaluation of targeted and systematic biopsies using magnetic resonance and ultrasound image-fusion guided transperineal prostate biopsy in patients with a previous negative biopsy. BJU Int 2017;120:631-638.

17. Tonttila PP, Lantto K, Pääkkö $E$, et al. Prebiopsy multiparametric magnetic resonance imaging for prostate cancer diagnosis in biopsy-naive men with suspected prostate cancer based on elevated prostate-specific antigen values: results from a randomized prospective blinded controlled trial. Eur Urol 2016;69:419-425.

18. Hofbauer SL, Maxeiner A, Kittner B, et al. Validation of Prostate Imaging Reporting and Data System Version 2 for the Detection of Prostate Cancer. J Urol 2018;200:767-773.

19. Mertan FV, Greer MD, Shih JH, et al. Prospective Evaluation of the Prostate Imaging Reporting and Data System Version 2 for Prostate Cancer Detection. J Urol 2016;196:690-696.

20. NiMhurchu E, O'Kelly F, Murphy IG, et al. Predictive value of PI-RADS classification in MRI-directed transrectal ultrasound guided prostate biopsy. Clin Radiol 2016;71:375-380. 